\title{
A AVALIAÇÃO DA REDE VENOSA PELA ENFERMAGEM EM MULHERES COM CÂNCER GINECOLÓGICO DURANTE O TRATAMENTO QUIMIOTERÁPICO
}

Venous network assessment by nursing in women with gynecological cancer during chemotherapy treatment

\author{
Evaluación de la red venosa por la enfermería en mujeres con cáncer ginecológico \\ durante el tratamiento de quimioterapia
}

Cristiane Regina Soares ${ }^{1}$

Ana Maria de Almeida²

Thais de Oliveira Gozzo ${ }^{3}$

\section{RESUMO}

Estudo de abordagem exploratória e descritiva que teve como objetivos: avaliar a rede venosa das mulheres com câncer cérvico uterino, no início e ao final do tratamento quimioterápico; analisar a ocorrência de flebite provocada pelas drogas utilizadas nos protocolos de quimioterapia neoadjuvante e adjuvante e relacionar os tipos de veia com os dispositivos mais utilizados, tempo de permanência e intercorrências. Utilizou-se um instrumento de avaliação da rede venosa para os membros superiores. Foram incluídas 20 mulheres atendidas em um hospital de ensino do interior do Estado de São Paulo. A avaliação da rede venosa demonstrou poucas alterações, e a intercorrência mais frequente foi o hematoma (60\%). Os resultados deste estudo apontam para aspectos da prática de enfermagem relacionados à administração de quimioterápicos e ressaltam a necessidade de elaborar e implantar protocolos para o cuidado.

Palavras-chave: Neoplasias do colo do útero. Enfermagem. Quimioterapia. Extravasamento de materiais terapêuticos e diagnósticos.

\begin{abstract}
This descriptive and exploratory study aimed to evaluate the venous network of women with cervical uterine cancer, at the beginning and at the end of the chemotherapy treatment; to analyze the occurrence of phlebitis caused by the drugs used in protocols of neoadjuvant and adjuvant chemotherapy and to relate the types of vein with the most used devices, length of stay and complications. An instrument was used to evaluate the venous network for upper limbs. Participants were twenty women who received care at a teaching hospital in the interior of the state of Sao Paulo. The evaluation of the venous network presented little changes and hematoma was the most frequent complication (60\%). The results point out aspects of the nursing practice, related to the administration of chemotherapeutic agents, and highlight the need to develop and implement care protocols.
\end{abstract}

Keywords: Cervix Neoplasm. Nursing. Chemotherapy. Extravasations of Therapeutic Materials and Diagnostic.

\section{Resumen}

Este estudio exploratorio y descriptivo tuvo como objetivos evaluar la red venosa de las mujeres con cáncer de cuello uterino en el comienzo y el final del tratamiento quimioterápico, analizar la ocurrencia de flebitis causada por los fármacos utilizados en los protocolos de quimioterapia neo adyuvante y adyuvante, y relacionar los tipos de venas con los dispositivos más utilizados, tiempo de permanencia y complicaciones. Se utilizó un instrumento para evaluar la red venosa de los miembros superiores. Participaron 20 mujeres tratadas en un hospital de enseñanza en el interior del estado de São Paulo. La evaluación de la red venosa mostró pocas alteraciones y la complicación más frecuente fue el hematoma (60\%). Los resultados del estudio apuntan aspectos de la práctica de enfermería relacionados a la administración de agentes quimioterápicos y señalan la necesidad de desarrollar e implementar protocolos de atención.

Palabras clave: Neoplasmas del Cuello Uterino. Enfermería. Quimioterapia. Extravasación de Material Terapéuticos y Diagnóstico.

\footnotetext{
${ }^{1}$ Aluna de graduação da Escola de Enfermagem de Ribeirão Preto da Universidade de São Paulo (EERP - USP). Ribeirão Preto-SP. Brasil. E - mail: cristiane.soares@usp.br. Enfermeira, Professora Associada da Escola de Enfermagem de Ribeirão Preto da Universidade de São Paulo (EERP-USP) Departamento de Enfermagem Materno-Infantil e Saúde Pública. Ribeirão Preto-SP. Brasil. E - mail: amalmeid@eerp.usp.br; ${ }^{3}$ Enfermeira, Professora Doutora da Escola de Enfermagem de Ribeirão Preto da Universidade de São Paulo (EERP-USP) Departamento de Enfermagem Materno-Infantil e Saúde Pública. Ribeirão Preto-SP. Brasil. E - mail: thaisog@eerp.usp.br.
} 


\section{INTRODUÇÃO}

A manipulação e a utilização de acessos venosos são práticas do cotidiano de diversas categorias de profissionais da saúde. A equipe de enfermagem utiliza veias para viabilizar terapêutica medicamentosa por períodos de curta ou longa duração, como a quimioterapia e antibioticoterapia, para investigação diagnóstica ou ainda para monitorização hemodinâmica do indivíduo'.

A punção venosa periférica (PVP) é um procedimento em que a pele do paciente é puncionada com uma agulha que irá permitir a inserção de um dispositivo na veia².

$\mathrm{Na}$ atualidade, o material utilizado para a punção venosa também é variado. Os dispositivos endovenosos para inserção periférica são materiais cilíndricos, canulados e perfurantes destinados a viabilizar a coleta de sangue e/ou a infusão de soluções. Muitos desses dispositivos são conhecidos pelo nome do fabricante, como scalp, dispositivo periférico de agulha rígida que possui haste extensora flexível, abocath ou jelco, um dispositivo venoso de agulha flexível sem haste, associado ao mandril, que deslizam no interior da agulha flexível ${ }^{1}$. Além disso, podem variar de acordo com o diâmetro da agulha, sendo mencionado 0 calibre, o número pelo qual são identificados no mercado consumidor e pelos profissionais da área da saúde.

A finalidade e o tempo previsto para o uso de um vaso sanguíneo podem influenciar na escolha do vaso, tipo de dispositivo intravenoso e sua fixação. As veias superficiais e as artérias dos membros superiores (MMSS) são as mais utilizadas para fins terapêuticos ${ }^{1}$.

Os adesivos, que são estruturas teciduais, podem ser sintéticos, semissintéticos ou naturais de dupla face, uma delas podendo ser impermeável e a outra conter cola para facilitar a adesão na superfície desejada'.

Os vasos puncionados para coleta de material ou administração de soluções podem sofrer danos. A ocorrência varia de acordo com o sítio da punção, tempo de permanência do acesso venoso e a forma como o dispositivo é retirado. Os danos podem ocorrer por transfixação do vaso, processos inflamatórios desenvolvidos por reação ao material empregado, extravasamento de sangue, que pode gerar equimoses e hematomas, e extravasamento de líquidos que estão sendo infundidos, podendo gerar desde edema até necrose tecidual'.

Além do aspecto técnico do material mais adequado para a PVP, é preciso considerar as características individuais de cada paciente. Deve ser realizada a avaliação da rede venosa, analisando as vantagens e desvantagens dos diferentes locais para a punção. Além disso, deve-se considerar o "medo de agulha" que o paciente pode ter, que causa vasoconstrição e dificulta o acesso venoso; a idade, em seus extremos, leva à fragilidade da pele e das veias; características do tecido no local da punção, como edema e obesidade, dificultam o procedimento, assim como o estado mental confus $0^{3}$.
Existem ainda fatores que limitam o número de veias disponíveis para punção, como fístulas para realização de hemodiálise, mulheres submetidas à cirurgia para 0 tratamento do câncer de mama, alergias, rash, psoríase, cicatrizes, radioterapia local, entre outras ${ }^{3}$.

Para o tratamento de pessoas com câncer, frequentemente há a necessidade de estabelecer um acesso venoso para administração dos agentes quimioterápicos, hemoderivados, antibióticos, nutrição parenteral, administração de fluidos e reposição de eletrólitos, além da coleta de sangue para exames.

A via endovenosa é a mais utilizada para a infusão de quimioterápicos, pois é considerada mais segura para a absorção e níveis séricos da droga, porém requer cuidados. Para uma administração segura, a enfermeira deve conhecer o tipo de droga que está infundindo, identificando se sua ação é vesicante ou irritante, o que requer cuidados no manejo adequado a partir das características da droga ${ }^{4}$.

A administração dos quimioterápicos por PVP pode estar relacionada a complicações como flebite, urticária, dor, eritema, descoloração ou hiperpigmentação venosa e necrose tecidual, secundária ao extravasamento ${ }^{4}$.

Além disso, existem os riscos adicionais relacionados à PVP dependendo do tempo de permanência da punção no mesmo sítio, sendo o mais comum a flebite, caracterizada por rubor e calor local, edema e formação de cordão fibroso palpável ao longo do trajeto venoso ${ }^{5}$.

A enfermeira e outros profissionais que integram a equipe oncológica devem ter conhecimento aprofundado sobre a quimioterapia usada no tratamento do câncer, além de informações precisas quanto à dosagem, esquema de aplicação e a toxicidade das combinações de drogas, para garantir a segurança do paciente e a qualidade do cuidado prestado.

Diante disso, este estudo teve como objetivos avaliar a rede venosa das mulheres com câncer ginecológico, no início e ao final do tratamento quimioterápico; analisar a ocorrência de flebite provocada pelas drogas utilizadas nos protocolos de quimioterapia neoadjuvante e adjuvante e relacionar os tipos de veia com os dispositivos mais utilizados, tempo de permanência e intercorrências, como flebite, extravasamento, infiltração e número de punções.

\section{MÉTODOS}

Estudo prospectivo, descritivo e exploratório, realizado no Hospital das Clínicas da Faculdade de Medicina de Ribeirão Preto da Universidade de São Paulo (HCFMRP - USP), na Unidade do Campus Universitário, na enfermaria de Ginecologia.

Por envolver seres humanos, o projeto de pesquisa foi submetido ao Comitê de Ética em Pesquisa do HCFMRP - USP, 
conforme parecer n⿳0 3170/2009, em cumprimento à Resolução CNS 196/96, tendo sido aprovado.

Foram incluídas mulheres atendidas no referido serviço de saúde que preencheram os critérios de inclusão: idade superior a 18 anos, primeiro tratamento quimioterápico para câncer ginecológico e uso dos esquemas quimioterápicos que necessitaram de internação por mais de dois dias.

Os protocolos de tratamento para a quimioterapia em uso no serviço em que o estudo foi realizado são paclitaxel, ifosfamida e cisplatina (TIP), cisplatina, ifosfamida e docetaxel (DIP) e vincristina, dactomicina e ciclofosfamida (VDC).

A coleta de dados foi realizada no período de junho de 2009 a maio de 2010, e utilizou-se um instrumento de avaliação da rede venosa para os membros superiores. 0 instrumento continha itens de avaliação da rede venosa e era aplicado antes de iniciar a infusão dos quimioterápicos e antes da alta hospitalar.

Para a avaliação da rede venosa foram seguidos critérios de visibilidade, palpação, mobilidade, calibre, trajeto e elasticidade, e a observação das punções venosas periféricas quanto aos locais, os dispositivos utilizados, o material para fixação do dispositivo, o principal motivo para a troca da punção, o tempo de permanência do cateter e as medicações administradas através da punção. 0 instrumento continha também informações sobre dados sociodemográficos, referentes ao diagnóstico de câncer e ao tratamento quimioterápico proposto.

Aanálise dos dados foi feita mediante estatística descritiva.

\section{RESULTADOS}

Foram incluídas 21 mulheres; entretanto, durante 0 tratamento, uma participante foi excluída do estudo por indicação de colocação de cateter totalmente implantado. Assim, a amostra foi composta por 20 mulheres. Todas elas tinham o diagnóstico de câncer cérvico uterino, $50 \%$ no estádio III e somente $5 \%$ em fase mais precoce da doença, estádio IB (Tabela 1).

A idade das participantes variou de 37 a 75 anos, sendo que $55 \%$ delas estavam na faixa etária de 51 a 60 anos; $50 \%$ eram casadas; $65 \%$ não completaram o ensino fundamental e $45 \%$ dedicavam-se ao lar (Tabela 1).

Tabela1: Distribuição das mulheres submetidas à quimioterapia para câncer de colo uterino, segundo idade, cor, escolaridade, estado civil, ocupação e estadiamento. Ribeirão Preto, $2010(\mathrm{~N}=20)$.

\begin{tabular}{lll}
\hline Características & Número e porcentagem \\
\hline Idade & & \\
31 a 40 anos & 3 & $15 \%$ \\
41 a 50 anos & 2 & $10 \%$ \\
> 50 anos & 15 & $75 \%$ \\
Cor & & \\
Branca & 14 & $70 \%$ \\
Negra e parda & 6 & $30 \%$ \\
Escolaridade & & \\
Ensino fund. Incom.e completo & 16 & $80 \%$ \\
Ensino médio Incom.e completo & 4 & $20 \%$ \\
Estado civil & & \\
Solteira/separada/viúva & 10 & $50 \%$ \\
Casada & 10 & $50 \%$ \\
Ocupação & & \\
Dona de casa & 9 & $45 \%$ \\
Lavradora & 2 & $10 \%$ \\
Doméstica & 6 & $30 \%$ \\
Outros & 3 & $15 \%$ \\
Estadiamento & & \\
IB1 & 1 & $5 \%$ \\
IIB & 9 & $45 \%$ \\
IIIA & 3 & $15 \%$ \\
IIIB & 7 & $35 \%$ \\
\hline
\end{tabular}


0 tratamento quimioterápico indicado para $95 \%$ mulheres foi o neoadjuvante e para 10\%, o adjuvante. Entre os protocolos utilizados, $60 \%$ das mulheres utilizaram o TIP, 35\% trataram com DIP e $5 \%$ delas com VDC. Entre as drogas, a vincristina e a dactinomicina são vesicantes.

Das 20 mulheres estudadas, $50 \%$ realizaram os três ciclos programados, uma delas realizou tratamento adjuvante e nove, o neoadjuvante. Para as que interromperam o tratamento, os motivos incluíram a ocorrência de reação de sensibilidade e ausência de resposta da doença ao tratamento quimioterápico.
A avaliação da rede venosa foi realizada antes do início de cada ciclo de quimioterapia e ao final deles, em todos os ciclos. Assim realizaram-se 20 avaliações no início do tratamento, com identificação das seguintes características da rede venosa: $100 \%$ veias visíveis; $80 \%$ veias palpáveis; $70 \%$ fixas; $65 \%$ veias com calibre médio; $80 \%$ veias com trajeto retilíneo e $95 \%$ veias flexíveis (Tabela 2).

Ao final dos três ciclos de tratamento programados, as 10 mulheres que os concluiu apresentaram rede venosa como descrito a seguir: $60 \%$ veias visíveis; $70 \%$ veias palpáveis; $70 \%$ fixas; $50 \%$ veias com calibre médio; $80 \%$ veias com trajeto retilíneo e $90 \%$ veias flexíveis (Tabela 2).

Tabela 2: Distribuição das mulheres submetidas à quimioterapia para câncer de colo uterino, segundo a avaliação da rede venosa ao início e ao final e as características da rede venosa. Ribeirão Preto, 2010 ( $N=20)$.

\begin{tabular}{lcc}
\hline Características da rede venosa & Início & Final \\
\hline Visibilidade & & \\
Veia visível & $100 \%$ & $60 \%$ \\
Veia de difícil visual ização & & $30 \%$ \\
Veia não visível & & $10 \%$ \\
Palpação & $80 \%$ & \\
Veia palpável & $15 \%$ & $70 \%$ \\
Veia não palpável & $5 \%$ & $30 \%$ \\
Veia sem condição de classificação & & \\
Mobilidade & $70 \%$ & $70 \%$ \\
Veia fixa & $30 \%$ & $20 \%$ \\
Veia móvel & & $10 \%$ \\
Veia sem condição de classificação & $25 \%$ & \\
Calibre & $65 \%$ & $40 \%$ \\
Pequeno & $10 \%$ & $50 \%$ \\
Médio & & $10 \%$ \\
Grande & $80 \%$ & $80 \%$ \\
Trajeto & $20 \%$ & $10 \%$ \\
Veia retilínea & & $10 \%$ \\
Veia tortuosa & & \\
Veia sem condição de classificação & $95 \%$ & $90 \%$ \\
Elasticidade & $5 \%$ & $10 \%$ \\
\hline Veia flexível & & \\
Veia endurecida & & \\
Veia sem condição de classificação & & \\
\hline & & \\
\hline
\end{tabular}

Durante o seguimento foram observados 138 sítios de punções venosas; os dispositivos mais utilizados foram o Saf-T-Intima $®$ com $63 \%$ das punções e abocath 20 ou 22 com 37\%. Para a fixação da punção venosa o material de escolha foi o esparadrapo, utilizado $83,3 \%$ vezes, seguido pelo evefix, em 11,5\%, e o micropore, em $5,8 \%$ das punções (Tabela 3 ).

Os locais para as punções venosas foram dorso da mão, com 66,7\% ocorrências e antebraço com $32(23,1 \%)$ e o membro superior com preferência para a realização das punções venosas foi o membro não dominante com 106 $(76,8 \%)$ punções e $32(23,1 \%)$ no membro dominante (Tabela 3).

Com relação ao número de tentativas por punção venosa, $33,3 \%$ das punções tiveram sucesso com apenas uma tentativa e $8 \%$, com duas ou mais tentativas no primeiro ciclo; $24,7 \%$ das punções foram realizadas com uma tentativa e $13,8 \%$, com duas ou mais tentativas no segundo ciclo de quimioterapia; $16 \%$ das punções foram realizadas com uma tentativa e $4,3 \%$, com duas ou mais no terceiro ciclo quimioterápico (Tabela 3).

Quanto ao tempo de permanência dos acessos venosos, considerado em horas, nos ciclos de quimioterapia, observou-se que o menor tempo foi de meia hora e o máximo, de 120 horas (Tabela 3). Os principais motivos para a troca da punção venosa durante os ciclos de quimioterapia foram por infiltração, perda acidental da punção e obstrução do cateter. 
Tabela 3: Distribuição das mulheres submetidas à quimioterapia para câncer de colo de útero, segundo as características das punções venosas. Ribeirão Preto, 2010 (N=20).

\begin{tabular}{lcc}
\hline Características & Número e porcentagem \\
\hline Dispositivo & \multicolumn{2}{c}{} \\
Saf-T-Intima ${ }^{\circledR}$ & 87 & $63 \%$ \\
Abocath & 51 & $37 \%$ \\
Fixação & & \\
Esparadrapo & 115 & $83,3 \%$ \\
Evefix & 16 & $11,5 \%$ \\
Micropore & 7 & $5,8 \%$ \\
Local & & \\
Dorso da Mão & 92 & $65,7 \%$ \\
Antebraço & 32 & $23,1 \%$ \\
Punho & 10 & $7,2 \%$ \\
Fossa anticubital & 4 & $2,9 \%$ \\
Membro Superior & & \\
Não Dominante & 106 & $76,8 \%$ \\
Dominante & 32 & $23,1 \%$ \\
Número de Tentativas & & \\
Uma & 102 & $73,9 \%$ \\
Duas & 21 & $15,2 \%$ \\
Três ou mais & 15 & $10,9 \%$ \\
\hline
\end{tabular}

Entre as intercorrências apresentadas, as mais frequentes foram o hematoma (60\%), a hiperemia ( $25 \%$ ) e relato de dor no local $(15 \%)$, e a principal conduta para cuidado em domicílio orientada pelos profissionais da saúde foi a utilização de compressas de chá de camomila e Hirudoid®

Não houve registro de extravasamento de drogas vesicantes, e foram identificadas $2,1 \%$ de infiltrações, sendo que somente um dos casos foi de quimioterápico (ifosfamida). As principais condutas dos profissionais diante da infiltração foram a retirada do acesso venoso em todos os casos, seguida de enfaixamento do local afetado ou uso de Hirudoid $@$. Não houve registro de complicações para as mulheres.

\section{DISCUSSÃO}

Apesar do número reduzido de participantes, a avaliação da rede venosa aponta para alterações nas características das veias no início e ao final do tratamento, destacando-se a redução na visibilidade, palpação, diminuição do calibre. Quanto à ocorrência de flebite, não houve nenhum registro.

Para a administração do tratamento quimioterápico é necessário estabelecer um acesso venoso periférico adequado. A literatura indica que se dê preferência para as veias mais calibrosas, menos tortuosas e móveis, distantes das articulações, evitando-se veias rígidas, endurecidas e com alterações de cor e doloridas. Alguns autores recomendam seguir uma ordem de preferência na escolha venosa: veias do dorso da mão, do punho e do antebraç ${ }^{4,8,9}$.

Essas condutas são recomendadas, pois frequentemente os pacientes oncológicos apresentam rede venosa de difíil punção evisualização. Podem ser responsáveis por essa precariedade venosa nesses pacientes a realização de múltiplas punções, trombocitopenias decorrentes do tratamento quimioterápico efragilidade capilar ocasionada pela própria agressividade do tratamento ${ }^{4,9,9}$.

A escolha bem-sucedida de uma veia depende diretamente do dispositivo utilizado, pois o calibre da veia e do dispositivo e o tipo de medicamento que vai ser infundido influenciam na realização da punção venosa. Os mais indicados para infusão de agentes quimioterápicos são os dispositivos flexíveis, pois eles são mais resistentes ao risco de deslocamento do dispositivo decorrente da movimentação do membro puncionado ${ }^{4}$, fato observado neste estudo.

Para a fixação do dispositivo, o mais utilizado neste estudo foi o esparadrapo; entretanto, o mais indicado é o filme adesivo transparente. Este material favorece a visualização do sítio de punção pelos profissionais da saúde e a detecção precoce de possíveis reações locais ${ }^{4,8}$. Entretanto, para uma fixação segura, independente do material escolhido, também se recomenda não colocar adesivos em excesso para não prejudicar a visibilidade da área de inserção do cateter $^{4,8}$.

A escolha do local da punção venosa periférica deve ser sempre nos membros superiores, iniciando da parte distal para a proximal do membro escolhido. Isso é indicado para que ocorra uma utilização mais racional dos vasos sanguíneos. Porém, áreas de articulação e flexão requerem estabilização, pois é relatado que o movimento irá ocorrer naturalmente quando são executadas atividades da vida diária; isso pode implicar uma possível perda da veia $a^{4,8,10-12}$.

Entre as principais condutas para a realização de punção venosa para administração do tratamento quimioterápico, é preconizada a escolha de veia periférica no membro superior, evitando-se punção de membros edemaciados, com lesões, com distúrbios motores ou sensoriais ou recentemente puncionados. 
Quanto ao dispositivo, são indicados os flexíveis, como o jelco e Saf-T-Intima $®$, e, para a fixação, indicam-se materiais que não prejudiquem a visibilidade da área puncionada ${ }^{4,8,9,14,15}$.

Outros fatores que devem ser considerados durante a infusão de agentes quimioterápicos são a certificação do correto posicionamento do dispositivo e do retorno venoso antes de aplicar a droga; manter a área puncionada sob observação constante durante o período da infusão; instruir o paciente a relatar imediatamente qualquer anormalidade, como dor, queimação, formigamento ou prurido; e documentar, nos prontuários do paciente todas as condutas e orientaç̃̃es realizadas antes, durante e após a infusão dos quimioterápicos $4,8,9,14,15$.

Com relação ao número de tentativas para a realização das punções venosas, é evidente que, na maioria, houve apenas uma tentativa. Isso é positivo, pois proporciona tranquilidade para as mulheres, preservação da rede venosa, diminuição dos riscos de infiltração, segurança ao profissional e qualidade da assistência de enfermagem ${ }^{4}$.

Quanto ao tempo de permanência do acesso venoso, a recomendação é de troca a cada 72 horas quando utilizado para infusão de soros e medicações e de 24 horas quando utilizado para administração de agentes quimioterápicos. Estes prazos podem ser reduzidos em casos de complicações no local da punção, como flebite, hematomas, infiltração ou extravasamento. Em casos, em que a punção venosa permanece superior a esse tempo, podem ocorrer como consequências traumas vasculares, devidos à irritação dos quimioterápicos ${ }^{4,11-13}$.

Diante de trauma vascular, as principais condutas e orientações da equipe de enfermagem foram a utilização de compressas de chá de camomila e do Hirudoid@ $@$ na região afetada. Entretanto, na literatura, as recomendações são mais específicas para drogas quimioterápicas classificadas como vesicantes, não havendo protocolos ou recomendações para outros medicamentos ${ }^{4,9,10,12,14 .}$

Diante disso, salientamos a importância da equipe de enfermagem em realizar pesquisas e construir instrumentos que possam auxiliar a sistematização da assistência de enfermagem às mulheres durante a quimioterapia. Um estudo realizado com 20 pacientes com câncer de pulmão em tratamento quimioterápico propõe um instrumento de coleta de dados que contempla dados gerais, requisitos de autocuidado universal e o exame físico, e os resultados apontam que ele pode ser um guia para a coleta de dadose, consequentemente, auxiliar nas práticas de enfermagem ${ }^{15}$. Em futuras pesquisas, pode-se ainda contemplar a avaliação da rede venosa, visto que o acesso venoso é o principal local de infusão dos quimioterápicos.

\section{CONSIDERAÇÕES FINAIS}

Apesar das limitações deste estudo em relação ao número de participantes, fato que inviabilizou análises estatísticas, os resultados apontam para aspectos da prática de enfermagem relacionados à administração de quimioterápicos e ressaltam a necessidade de elaborar e implantar protocolos para o cuidado. Estes protocolos devem abranger desde os procedimentos mais simples, como a escolha do acesso venoso, materiais a serem utilizados na punção e fixação, até os mais complexos, como identificação de intercorrências e o manejo destas.

Além disso, os enfermeiros devem ser encorajados a realizar pesquisas científicas para identificar estratégias de prevenção e intervenção mais efetivas no controle e manejo das possíveis intercorrências relacionadas à rede venosa de pacientes em tratamento quimioterápico, buscando melhorar a qualidade de vida do paciente e valorizar o trabalho do enfermeiro.

\section{REFERÊNCIAS}

1.Arreguy-Sena C. A trajetóría de construção e validação dos diagnósticos de enfermagem: trauma vascular erisco paratraumavascular [tese de doutorado]. Ribeirão Preto: Escola de Enfermagem/USP); 2002.

2.Jackson A. Reflecting on the nursing contribution to vascular access. Br J Nurs. 2003; 12 (11): 657-65.

3.Wells S. Venous access in oncology and hematology patients: par tone. Nurs Stand. 2008; 22 (52): 39-46.

4.Bonassa EMA. Enfermagem em terapêutica oncológica. São Paulo: Atheneu; 2005.

5.Macklin D. Phlebitis a painful complication of peripheral IV catheterization that may be prevented. Am J Nurs. 2003; 103 (2): 55-60.

6. Instituto Nacional do Câncer-INCA. [site de internet]. Estimativa 2010: incidência de câncer no Brasil. [citado 2009] Disponível em: http:///umwinca.gov.br.

7.Instituto Nacional do Câncer-INCA. [site de internet]. Ações de enfermagem para o controle do câncer: uma proposta de integração ensino-serviço. [citado 2008] Disponível em: http://umwinca.gov.br.

8. Hadaway L. Infiltration and extravasation preventing a complication of IV catheterization. Am J Nurs. 2007; 107 (8):64-72.

9.Doellman D, Hadaway L, Geddes-Bowe LA, Franklin M, Leddone J, PapkeO'Donell L, et al. Infiltration and extravasation update on prevetion and management. I Infus Nurs. 2009; 32 (4): 203-10.

10.Wengström Y, MarguliesA. European oncologynursing society extravasation guidelines. Eur J Oncol Nurs. 2008; 12 (4): 357-61.

11.Sauerland C, Engelking C, Wickhan R, Corbi D. Vesicant extravasation part l: mechanisms, pathogenesis, and nursing care to reduce risk. Oncol Nurs Forum. 2006; 33(6): 1134-41.

12.Thim IC. Peripheral intravenous catheters: considerations in theory and practice. $\mathrm{Br} J$ Nurs Scales K. Intravenous therapy: a guide to good practice. Br J Nurs. 2008; 17 (19): 4-12 
A avaliação da rede venosa em mulheres com câncer ginecológico

Soares CR, Almeida AM, Gozzo TO

13.Adami NP, Gutíerrez MGR, Fonseca SM, Almeida EPM. Risk management of extravasation of cytostatic drugs at the adult chemotherapy outpatient clinic of a university hospital. Cancer Nurs. 2005; 14: 876-82.

14.Schulmeister L. Vesicant chemotherapy -the management of extravasation. Cancer Nurs Pract. 2009; 8 (3): 34-7.

15.SalvadoriAM, Lamas JLT,Zanon C. Desenvolvimento de instrumento de coleta dedados de enfermagem para pacientes com câncer de pulmão em quimioterapia ambulatorial. Esc Anna Nery. 2008; 12(1): 130-35. 